\title{
Correction to: A double-blind placebo controlled study of acotiamide hydrochloride for efficacy on gastrointestinal motility of patients with functional dyspepsia
}

\author{
Kumiko Nakamura $^{1}$ - Toshihiko Tomita ${ }^{1}$ Tadayuki Oshima ${ }^{1} \cdot$ Haruki Asano ${ }^{1}$. \\ Takahisa Yamasaki $^{1}$ - Takuya Okugawa ${ }^{1}$ - Takashi Kondo ${ }^{1}$. Tomoaki Kono ${ }^{1}$ • \\ Katsuyuki Tozawa $^{1} \cdot$ Yoshio Ohda $^{1} \cdot$ Hirokazu Fukui ${ }^{1} \cdot$ Fukushima Kazuhito $^{2}$. \\ Shozo Hirota $^{2} \cdot$ Jiro Watari $^{1} \cdot$ Hiroto Miwa ${ }^{1}$
}

Published online: 25 January 2021

(C) Japanese Society of Gastroenterology 2021

\section{Correction to: J Gastroenterol (2017) 52:602-610 https://doi.org/10.1007/s00535-016-1260-7}

In the original publication of the article, the following description was missing in the COI disclose section and it has been given in this correction.
Conflict of interest Miwa $\mathrm{H}$ received commercial research funding from Astellas pharmaceutical company.

The original article can be found online at https://doi.org/10.1007/ s00535-016-1260-7.

\section{Hiroto Miwa}

miwahgi@hyo-med.ac.jp

1 Division of Gastroenterology, Department of InternalMedicine, Hyogo College of Medicine, 1-1, Mukogawa-cho, Nishinomiya, Hyogo 663-8501, Japan

2 Department of Radiology, Hyogo College of Medicine, Nishinomiya, Hyogo, Japan 